Revista de Derecho

\title{
Cambios tecnológico-productivos del trabajo: problemática jurídica ecuatoriana
}

\section{Technological-Productive Changes in Work:} Ecuadorian Juridical Issues

\author{
Carolina Jacqueline Rodríguez Mendoza \\ Docente de la Universidad Regional Autónoma de los Andes \\ Tulcán, Ecuador \\ ut.carolinarm16@uniandes.edu.ec \\ ORCID: 0000-0002-2001-5264
}

\section{Milton Enrique Rocha Pullopaxi}

Docente de la Universidad Indoamérica

Quito, Ecuador

miltonerri@gmail.com

ORCID: 0000-0001-7871-6613

Artículo de investigación

DOI: https://doi.org/10.32719/26312484.2022.37.6

Fecha de recepción: 11 de mayo de 2021

Fecha de revisión: 11 de agostor de 2021

Fecha de aceptación: 15 de septiembre de 2021

Fecha de publicación: 3 de enero de 2022 


\section{RESUMEN}

Este estudio tuvo por objetivo esquematizar las relaciones entre las transformaciones tecnológicas y productivas en el ámbito laboral. Para cumplir con este fin, se siguió una metodología cualitativa de revisión documental y de aporte intuitivo sobre la base teórico descriptiva de conceptos como: tecnología, innovación, creación, cambios sociales, transformaciones productivas, y, principalmente, empleo en el marco del concepto trabajo. Al considerar el nivel descriptivo y exploratorio, se delinean las relaciones que se han encontrado en la literatura científica en esta área del conocimiento y se formulan la posible existencia de más relaciones. Finalmente, este escrito, expone la importancia del cambio tecnológico; mira el proceso de transformación tecnológica en el contexto social, plantea la emergencia o necesidad de un nuevo paradigma; fija la relación entre tecnología, innovación tecnológica, cambio social y trabajo para concluir sobre sus dimensiones, el tipo de relación y sus efectos; también pone de manifiesto las dificultades, límites y retos que representa este tema para el mundo contemporáneo, especialmente sobre el empleo desde una perspectiva jurídica ilustrada en problemáticas del contexto actual ecuatoriano.

Palabras Clave: trabajo; transformaciones; tecnológico; productividad; jurídico; Ecuador.

\section{ABSTRACT}

The research objective has been to outline the relationships between technological and productive transformations in the work. To reach that, a qualitative methodology of documentary review and intuitive contribution was followed on the theoretical descriptive basis of concepts such as: technology, innovation, creation, social changes, productive transformations; and, mainly, employment within the framework of the concept of work. As it is a descriptive and exploratory level investigation; The relationships that have been found from the scientific literature in this area of knowledge are delineated and the possible existence of more relationships is formulated. Finally, this study exposes the importance of technological change; look at the process of technological transformation in the social context, raises the emergency or need for a new paradigm; fixes the relationship between technology, technological innovation, social change and work to conclude on its dimensions, the type of relationship and its effects; It also highlights the difficulties, limits and challenges that this issue represents for the contemporary world, especially on employment from a legal perspective illustrated in problems of the current Ecuadorian context.

KEYWORDS: work; transformations; technological; productivity; legal; Ecuador. 


\section{INTRODUCCIÓN}

$\mathrm{E}$ 1 presente trabajo de investigación trata el fenómeno social de los cambios tecnológicos y productivos en el ámbito laboral, siendo indispensable su estudio debido a que es una constante del mundo contemporáneo, y ha tenido un crecimiento acelerado en las últimas décadas.

La idea central de este artículo consiste en establecer una aproximación descriptiva de las relaciones que suceden entre las transformaciones tecnológicas y productivas en un contexto laboral o de trabajo desde una perspectiva jurídica que asume las problemáticas de la actual realidad ecuatoriana.

La importancia y la relevancia de la temática resaltan porque su incidencia cubre todas las esferas de la vida en sociedad, sea pública o privada, y hasta las relaciones con el entorno-naturaleza, por lo cual un acercamiento desde una perspectiva jurídica es abonar a este amplio espectro de conocimiento.

Las aportaciones que se desarrollan en este trabajo se pueden verificar en tres segmentos: 1) Aportaciones teóricas, debido al establecimiento de las relaciones existentes entre las categorías estudiadas y de sumar una aproximación ilustrativa desde el ámbito jurídico al fenómeno del cambio tecnológico; 2) Aportaciones metodológicas, en razón del planteamiento descriptivo y exploratorio que permiten incrementar el conocimiento - más relaciones entre categorías - y desde distintas disciplinas; y 3) Utilidad práctica o técnica, aunque no genere instrumentos o herramientas, sí sienta las bases para que puedan elaborarse.

La línea argumentativa para el desarrollo de contenidos se construye desde cuatro ejes centrales: 1) Los cambios tecnológicos hacen necesario que se produzca un nuevo paradigma sobre el proceso de transformación tecnológica multidimensional; 2) La relación entre cambios tecnológicos y cambios sociales - incluyendo cambios culturales - es bidireccional como indicador de avance entre la idea de cambio-innovación-cambio; 3) La relación anterior sucede con mayor presencia en un contexto de trabajo; y, mucho más específico, en lo laboral y el empleo; por lo tanto, es imprescindible abordar una segunda relación entre transformaciones tecnológicas y productivas en situaciones de trabajo típico; y 4) Las problemáticas pueden ser múltiples entorno a estas relaciones en el ámbito laboral, no obstante, más notorias desde una perspectiva jurídica en situaciones de trabajo atípico.

La confirmación de argumentos permitirá llegar al objeto de determinación exploratoria y descriptiva de la problemática jurídica que resulta del establecimiento de las relaciones entre cambios tecnológicos y productivos del trabajo, sin que necesariamente entre a una reflexión profunda dentro del ámbito jurídico, sino que ponga de manifiesto posibles escenarios en los que representa una problemática. 
En los primeros cinco apartados se encontrará la construcción teórica y determinación de relaciones de las categorías enunciadas; mientras que el sexto hará un ejercicio de exploración ilustrativa de la problemática jurídica dejando a salvo reflexiones profundas para futuros trabajos.

\section{CAMBIO TECNOLÓGICO}

La producción de tecnología es un proceso que busca dar solución a problemas de carácter simple o complejo, que no solo incluye incorporación de máquinas o equipos, también involucra aplicación de conocimientos prácticos y teóricos, que ante los desafíos de cada etapa buscan generar nuevas formas de producción, organización y comercialización que respondan a los patrones de consumo. ${ }^{1}$

El cambio tecnológico presenta elementos distintivos asociados al conocimiento como: factor productivo, innovación como base para mantener una posición ventajosa y diferencial en el mercado y el desarrollo sistemas que permiten una mayor conexión entre las personas. ${ }^{2}$ Esto ha facilitado la globalización de los mercados e impulsado nuevas modalidades productivas.

Con la cuarta revolución industrial se han profundizado "el desarrollo de aplicaciones en el campo de la cibernética y de las tecnologías de información y comunicación". 3 Avances tecnológicos que permiten automatizar tareas cognitivas, controlar procesos físicos, analizar y extraer información del mundo virtual.

\section{TRANSFORMACIÓN TECNOLÓGICA EN LO SOCIAL: NUEVO PARADIGMA}

Toda transformación que se presenta en el plano del conocimiento y de la realidad concreta genera impactos en varias dimensiones — social, económica, cultural, política, etc. - de donde surgen varios tipos de relaciones. Para comprender esta constelación de conocimientos de manera sistematizada es necesario plantear la posibilidad de un paradigma sobre el proceso de transformación tecnológica que, según Lück,

1. Sonia Roitter, Cambio tecnológico y empleo: Aportes conceptuales y evidencia frente a la dinámica en curso (Buenos Aires: Centro Interdisciplinario de Estudios en Ciencia, Tecnología e Innovación, 2019), 17.

2. Ibíd., 22.

3. Ana Catalano, Tecnología, innovación y competencias ocupacionales en la sociedad del conocimiento, Documentos de Trabajo 22 (Argentina: Oficina de País de la OIT para la Argentina, 2018), 18, https://url2. $\mathrm{cl} / \mathrm{MtVcH}$. 
basado en Castelles, tiene algunas características que representan la base material de la sociedad de la información: ${ }^{4}$

Primera, la información se constituye en la materia prima de la tecnología y no a la inversa; por lo tanto, es parte sustancial del nuevo paradigma tecnológico que simplifica la información con influencia sobre la tecnología. Segunda, la tecnología se incorpora en todas las esferas de la vida humana de manera individual o colectiva. Tercera, la información como fenómeno complejo y de gran interacción a través de la tecnología puede entenderse desde la morfología de la red.

Cuarta, no es necesario destruir una institución u organización cuando existe una flexibilidad del sistema de redes, es decir, se puede modificar, configurar, reconfigurar, programar y reprogramar procesos sin que esto signifique una desaparición de la base material de la organización. Quinta, convergencia de la tecnología con un sistema de información integrado, por ejemplo, tecnología específica para un área de conocimiento, por ejemplo, estudios neurolingüísticos a través de Resonancia Magnético Nuclear (RMN) y computarizada.

Lück, agrega que "la economía informacional no se basa apenas en la información, pero sí en la inclusión de atributos culturales en la implementación y diseminación del nuevo paradigma tecnológico". ${ }^{5}$ Es decir, que las relaciones Estado-sociedad global; Estado-sociedad-nación; Estado-nacionalidades y pueblos; Estado-instituciones; y a la inversa y entre quienes componen el mundo como personas naturales y jurídicas a nivel individual o colectivo, nacional o internacional; tienen un rol importante en los procesos que involucran la tecnología y la toma de decisiones.

En ese sentido, existe una emergencia en la formulación de un paradigma tecnológico en vista de que la información-tecnología-decisión, muchas veces no se relacionan de manera adecuada; sobre todo la última, debido a que esta depende de la voluntad política, de la pertinencia normativa, de la administración y gestión de los estados, de los recursos asignados, del compromiso social, y los objetivos e ideales que se persiguen como una sociedad de un Estado determinado.

Para acercarse a la comprensión más adecuada de la relación entre las transformaciones tecnológicas y las productivas en el ámbito del trabajo es necesario plantear el esquema de la información y de la decisión a los niveles y dimensiones antes comentados; cuestión que no se podrá profundizar en este trabajo académico debido a la extensión del tratamiento, pero sí se hace referencia a la emergencia de su estudio.

4. Esther Hermes Lück, "El proceso de transformación tecnológica y la formación docente", Revista de Universidad y Sociedad del Conocimiento 6, n. ${ }^{\circ} 1$ (2009): 3, https://n9.cl/b0bw.

5. Ibíd., 3 . 


\section{INNOVACIÓN TECNOLÓGICA, CAMBIO SOCIAL Y TRABAJO}

En la anterior sección se puso de manifiesto que existen varios tipos de relaciones; y, en esa línea lógica, una relación especial sucede entre los cambios tecnológicos y los cambios sociales.

Para Bouza, la relación entre estos dos aspectos es de tipo bidireccional, es decir, que no necesariamente uno de los dos es el determinante de la relación. En otras palabras, los efectos que producen las transformaciones tecnológicas en los cambios sociales no son los únicos, sino que sucede también a la inversa. ${ }^{6}$ Ejemplo, si hay cambios sociales, se deriva un número considerable de demandas sobre la tecnología. Así, de manera cíclica, las creaciones o innovaciones tecnológicas impulsarán algunos cambios sociales.

Se produce también una idea cíclica de cambio-innovación-cambio. Por ello, la elaboración de conocimiento a tecnología es un indicador del avance como sociedad. Puede haber una presencia de innovación que genera mucho más grado de avance tecnológico; y, por lo tanto, impulsar el cambio social; como puede haber un grado de "innovación" algo repetitivo o redundante que no contribuye a este cambio social y, que por el contrario, lo estatiza o retrocede. ${ }^{7}$

En esa lógica también sucede una relación de cambios tecnológicos y cambios culturales. Podría decirse que "de las deficiencias del mercado de trabajo y de la nostalgia colectiva hacia el supuesto paraíso perdido proceden los aspectos más sombríos del aún vacilante cambio social en curso". ${ }^{8}$

En este punto del ensayo se comienza a dar la importancia a la variable "trabajo" como un eje medular entre la transformación tecnológica y productivas en el contexto de cambios sociales (entre ellos, culturales y políticos).

Para delimitar el campo de análisis en los siguientes apartados se irán haciendo consideraciones dirigidas a contextualizar las categorías y sus relaciones en un contexto de trabajo incluyendo una perspectiva jurídica.

\footnotetext{
6. Fermín Bouza, "Innovación tecnológica y cambio social”, en Las encrucijadas del cambio social, Universidad de Vigo (Vigo: Servizo de Publicacións, 2002), 85-97.

7. Ibíd., 91.

8. Ibíd., 96.
} 


\section{TRANSFORMACIONES TECNOLÓGICAS Y PRODUCTIVAS EN TORNO AL TRABAJO}

El trabajo, según la Real Academia Española, presenta varias acepciones como: a) verbo que consiste en la acción y efecto de trabajar; b) en el sentido de oficio se puede considerar una ocupación retribuida; c) como resultado o producto es una obra, algo que lo realizó un agente; d) manifestación de la actividad humana reflejada en una obra; e) operación de alguna máquina o herramienta con un fin determinado; entre otras, pero que sustancialmente la que más se liga a la idea que se pretende relacionar es la siguiente: "esfuerzo humano aplicado a la producción de riqueza, en contraposición a capital". ${ }^{9}$ Esta, aunque parece una acepción jurídica por el sentido de la contraprestación de la remuneración por una actividad laboral, pone especial énfasis en la idea de la producción de la riqueza.

Antes de empezar con el análisis de la relación de las transformaciones tecnológicas y productivas, es fundamental introducir elementos desde la normativa ecuatoriana sobre la definición de trabajo. La Constitución de la República del Ecuador de 2008 (en adelante, la Constitución) sostiene que el trabajo es un derecho, deber social, y derecho económico. ${ }^{10}$

El reconocimiento jurídico del trabajo presenta dos dimensiones: social y económica. Más allá de esto, se establece como fuente de realización personal y base de la economía; es decir, no solo forma parte de la vida jurídica, sino que resume mucho de lo que significa la humanidad y además un eje económico de las sociedades contemporáneas. Esta connotación histórica, filosófica y existencial podría resumirse en que sin trabajo no hay vida y sin el mismo no puede trascender las sociedades ni del mundo.

En ese orden de ideas, la Constitución en su artículo 319 plantea como relación sustancial el trabajo y la producción sintetizada en las formas de organización de la producción y su gestión. ${ }^{11}$ También se puede entender la relación del sentido de creación e innovación cuando se refiere a propiedad intelectual e industrial.

Otro de los elementos y relaciones que se reconocen son aquellas que se producen por el efecto del trabajo en la retribución y la redistribución de la riqueza. El primero, sobre retribución, porque para alcanzar una vida digna en el sentido individual y fa-

9. Real Academia Española, Diccionario de la lengua española (RAE, 2014), https://dle.rae.es sobre el término "trabajo".

10. Ecuador, Constitución de la República del Ecuador, Registro Oficial Suplemento 449, 20 de octubre de 2008, art. 33.

11. Ibíd., arts. 319-322. 
miliar la contraprestación garantiza que se cubra con las necesidades básicas; y, por lo tanto, la satisfacción de varios derechos. El segundo, la redistribución de la riqueza, enmarca el deber del Estado sobre la garantía y el desarrollo de políticas públicas manifestado prácticamente en los planes, programas, proyectos, entre otros, que ejecutan cada acción para la consecución de varios derechos.

Tanto normativa como política pública moldean en cada Estado y sociedad el ser y el deber ser del trabajo y de sus implicaciones en todas las esferas de la vida. A continuación, se expone una serie de elementos de análisis para la comprensión de la relación: transformaciones tecnológicas y productivas en el contexto del trabajo. Esto tiene una suerte de aplicación de sistemas que inciden sobre el empleo de una forma diferencial ya sea de manera sustitutiva o complementaria sobre las tareas y de forma negativa para los empleos caracterizados por tareas rutinarias. ${ }^{12}$

Desde el enfoque del mecanismo de compensación, los puestos de trabajo que son remplazados por la implementación de tecnología en la cadena de producción, pueden ser compensados considerando que los sistemas tecnológicos posibilitan una mayor producción con reducción de costos y precios lo que genera mayor demanda, y esto posibilita necesidad de nuevos puestos de trabajo para atender la necesidad emergente.

En efecto, Marx en su análisis sobre este tema señala que la incorporación de maquinarias aumenta la productibilidad, sin embargo, a diferencia del enfoque de “compensación", el trabajo humano será remplazado por el de la máquina y no generara puestos de trabajo, lo que se verá reflejado en aumento de las tasas de desempleo, considerando que la compensación es parcial a la velocidad con la que se da el crecimiento de la demanda no responde a la incorporación inmediata de los trabajadores desplazados. ${ }^{13}$

Según Roitter, desde la perspectiva de Petit, "la compensación no se produce en términos de empleo ya que, capital y trabajo se utilizarían hasta el punto en que ambos obtengan el mayor retorno marginal en términos de ganancias y utilidad, respectivamente, al igualar la productividad marginal por unidad de valor invertida en cada factor". ${ }^{14}$ En este sentido existe un punto de ajuste del mercado, la innovación tecnológica no genera desempleo y existe beneficio tanto para el trabajador como para el

12. Jeremy Rifkin, El fin del trabajo: Nuevas tecnologías contra puestos de trabajo (Nueva York: Paidós Ibérica, 1996).

13. Karl Marx, El Capital, traducido por Isaac Zeiger (Buenos Aires: Gradifco, Pensadores Universales, 2006).

14. Roitter, Cambio tecnológico y empleo: Aportes conceptuales y evidencia frente a la dinámica en curso, 31. 
empleador. Para Keynes esta perspectiva no toma en cuenta la baja demanda efectiva prediciendo que no existirá el equilibrio para mantener el empleo.

La incertidumbre nace del impacto del avance tecnológico, la capacidad de respuesta de los nuevos sistemas productivos para generar empleo y la cualificación de los trabajadores ante los nuevos requerimientos, el proceso de cambio plantea estos cuestionamientos en la medida de que no se tiene claro si la transformación por la que se está atravesando actualmente tenga la capacidad de generar el mismo número de empleos que va desplazando, por lo que se presentan una serie de perspectivas que se exponen a continuación:

Desde una visión radical, Ford señala que, a medida que avanza el desarrollo tecnológico, aumenta el riesgo sobre ocupaciones que pueden perderse como consecuencia de la automatización. ${ }^{15}$

Autores como Frey y Osborne, plantearon un análisis enfocado en la idea de que ciertas ocupaciones pueden ser computarizadas, y que en la medida que se presenta el avance tecnológico tiene un efecto negativo sobre el empleo, especialmente en actividades de construcción, logística, transporte y manufactureras. ${ }^{16}$

Algunos llegan al conceso de que los cambios tecnológicos tendrán un impacto negativo sobre las actividades rutinarias y que sus efectos también alcanzarán a las actividades cognitivas no rutinarias, en esta línea la destrucción no será solo para ocupaciones o tareas específicas, incluso se destaca la incidencia negativa de las nuevas tecnologías sobre el empleo a partir de los avances digitales que incrementan productividad sin requerir incorporación masiva de mano de obra.

Frente a estas perspectivas se presentan otras alternativas centradas en mecanismos de complementariedad antes que en los efectos de la sustitución, lo que invita a pensar en que este mecanismo posibilita la generación de empleos a partir de la necesidad de nuevas actividades.

En cierto modo, se sostiene que los cambios tecnológicos complementan los procesos de automatización en la medida que generan aumento de producción, $\mathrm{y}$, por ende, el requerimiento de mano de obra, y señalan que la disminución del empleo no es consecuencia de la innovación tecnológica, sino de la falta de capacidad para mejorar la productividad y el producto a partir de mejoras tecnológicas. A la par se

15. Martin Ford, El auge de los robots: La tecnología y la amenaza de un futuro sin empleo (Barcelona: Paidós Ibérica, 2016).

16. Carl Frey y Michael Osborne, "The future of employment: How susceptible are jobs to computerisation?", Technological Forecasting \& Social Change 49 (2017): 254-80, doi:https://url2.cl/5z9Rs. 
establece que la sustitución de trabajo se da principalmente en tareas rutinarias, y se mantiene que el trabajo cognitivo será difícilmente sustituido.

Con referencia a la creación de nuevos empleos se establece que el impacto tecnológico es gradual considerando que, en un primer momento, se destruyen ocupaciones, se modifican tareas existentes, cambia la organización y, a partir de esto, se genera la disminución de la masa laboral, lo que permite ajustes y cambios dando paso a la creación de puestos de trabajo; claro está que estos procesos van acompañados de requerimientos de competencias y cualificaciones de los trabajadores, que se deben ajustar al proceso de cambio.

Otro aporte importante son las particularidades de los tipos de trabajo y las ocupaciones; también las tareas y ocupación, si en las actividades que se realizan predomina el trabajo manual o cognitivo y el grado de rutinización que puede presentar cada una de ellas; esta distinción permitirá diferenciar el impacto que tendrá el cambio tecnológico sobre estos grupos.

En efecto, las tareas y ocupaciones en las que predomina el trabajo manual son propensas a ser codificadas y sistematizadas, también afecta a las actividades que en parte son rutinarias y cognitivas, en un menor grado en las actividades que requieren esfuerzo cognitivo, intuición, interacción, adaptabilidad y no son repetitivas, aunque no se descarta la idea de que las innovaciones tecnológicas permitan insertarse en el ámbito de las actividades cognitivas.

Para Arnzt, Gregory y Zierahn en algunos casos los procesos de automatización no destruyen labores, sino que el efecto se da sobre parte de las tareas de la ocupación, por ejemplo, las actividades específicas como la contabilidad, el trabajo administrativo y aquellos que son repetitivos en las fábricas por medio de la aplicación de sistemas informáticos se han ido remplazando. ${ }^{17}$

Lo que puede ser diferente en trabajos que requieren interacción social, por ejemplo, negociadores, vendedores, cuidadores de personas con altos niveles de dependencia o enfermas, entre otros, actividades que se caracterizan por aplicación de la inteligencia emocional, siendo difícil su automatización por su naturaleza de experiencia humana.

En términos de nivel de cualificación, algunos estudios coinciden en que el efecto negativo de la automatización se reflejará en aquellas actividades que requieren un ni-

17. Melanie Arntz, Gregory Terry, y Ulrich Zierahn, "The Risk of Automation for Jobs in oecd Countries: A Comparative Analysis", Employment and Migration Working Papers, n. ${ }^{\circ} 186$ (2016): 1-33, doi:https:// url2.cl/32ila. 
vel bajo de cualificación; ${ }^{18}$ y, por otra parte, existirían oportunidades para actividades debido a la imposibilidad de automatizar tareas basadas en la experiencia como en el caso mencionado en el párrafo anterior.

Otro análisis está encaminado a evaluar los horizontes laborales diferentes, considerando que la tecnología informática puede crear o cambiar empleos y estos requieren ciertos niveles de cualificación de los trabajadores para responder a los cambios y nuevas necesidades, especialmente relacionadas con la creatividad y habilidad para resolver problemas.

"Los cambios en las calificaciones y capacidades, así como los incrementos en la productividad que se derivan de las transformaciones tecnológicas recientes también tienen su impacto en los niveles de remuneración de los trabajadores", ${ }^{19}$ frente a la desaparición de ocupaciones de mediana cualificación, los empleos de estratos altamente cualificados serán los que presenten mejores niveles salariales.

Por un lado, tenemos los empleos que requieren habilidades especiales para resolver problemas ubicados en altos estratos cualificados y a la par los de estrato bajo poco cualificados que no pueden ser automatizados debido a que los sistemas tecnológicos no pueden reproducir su experiencia, o por las escasas productividad que representan ciertas actividades.

Desde otro ángulo, se hace una evaluación de los aportes de la tecnología frente a la organización del trabajo y la calidad del empleo, en este sentido las transformaciones tecnológicas favorecen a las técnicas de producción, comercialización y distribución.

La tecnología digital ofrece a las empresas la posibilidad de prescindir de ciertos puestos de trabajo, digitalizar tareas y reubicar puestos. La deslocalización permite evaluar costos de contratación de un país a otro, generando empleo en el país seleccionado y a la par permite el surgimiento de nuevas actividades de servicios orientados a la actividad industrial.

Son algunos de los ejemplos de las relaciones que suceden a las transformaciones tecnológicas y productivas sobre el trabajo y más en el empleo, en un sentido básico de hipótesis tentativa donde en varios casos la tecnología contribuye a la producción, pero no genera empleos; $y$, por otro lado, cuando sí lo hace en un contexto de trabajo

18. Abramo Lais y Salazar, "El trabajador frente a la automatización: efectos sociales y percepción de los trabajadores", Revista Mexicana de Sociología 50 (1988): 61-9, doi:10.2307/3540584; Daniel Martínez, "Sobre el incierto futuro del trabajo y del rol de los actores sociales", Revista Economía 41, n. ${ }^{8} 81$ (2018): 69-100, doi:https://url2.cl/sDI8y; Rafael Doménech et al., “Afectados por la revolución digital: El caso de España", Papeles de Economía Española, 2018, 128-45.

19. Roitter, Cambio tecnológico y empleo: Aportes conceptuales y evidencia frente a la dinámica en curso, 50 . 
típico, no obstante, queda en siguientes líneas formular de manera exploratoria si esto se repite de la misma manera en el trabajo atípico.

\section{TRANSFORMACIONES TECNOLÓGICAS Y PRODUCTIVAS: TRABAJO ATÍPICO}

Un mundo interconectado determina nuevos patrones de comportamiento, y promueve nuevas estructuras organizacionales adaptadas para minimizar costos y facilitar transacciones, lo que cambia la dinámica del mercado de consumo pues existe una gran variedad de actividades, bienes y servicios que son ofertados a través de plataformas digitales, es precisamente este contexto el que determina el surgimiento de nuevas modalidades de trabajo. ${ }^{20}$

Los cambios tecnológicos marcan una transición del empleo formal a modalidades atípicas de empleo, por un lado el empleo formal se caracteriza entre otras cosas por ser continuo, estable, de tiempo completo, personal, bajo dependencia y con una remuneración, además de ofrecer a los trabajadores cierta estabilidad económica, sentido de pertinencia e integración social mientras que en el empleo atípico la jornada laboral es flexible, carece de estabilidad, no se genera vinculo de pertinencia, el trabajador es responsable de su propio esfuerzo respecto a la generación de ingresos y cuidado de su seguridad y salud.

Según informe de la OIT de 2016 las formas no estándar de empleo responden a arreglos sobre la jornada laboral, el tipo de contratación y la estabilidad del puesto de trabajo, entre ellas se distingue de manera general las siguientes categorías: a) empleo temporal por ser una modalidad de duración determinada, la actividad puede ser trabajo por proyectos o tareas; b) trabajo a tiempo parcial su jornada de trabajo es menor a 8 horas; c) trabajo a pedido, las horas de trabajo son reducidas y sus horarios tienen a ser poco predecibles; d) trabajo multipartita característico por su régimen de subcontratación; y e) empleo encubierto por cuenta propia económicamente dependiente. ${ }^{21}$

Estas categorías bien pueden trasladarse como ejemplificación en el uso de plataformas digitales que actualmente comienzan a cubrir el mercado tanto nacional como internacional; entre ellas, se puede citar Uber, Cabify, Globo, entre otros, que por sus características deben observase bajo criterios de primacía de la realidad, especial-

20. Javier Madariaga et al., Economía de plataformas y empleo ¿Cómo es trabajar para una app en Argentina? (Buenos Aires: CIPPEC-BID / OIT, 2009), 129.

21. OIT, "El empleo atípico en el mundo: Retos y perspectivas. Presentación resumida del informe" (Ginebra: Oficina Internacional del Trabajo, 2016), 2. 
mente la relación existente entre los sujetos y las condiciones para el desarrollo de la prestación del servicio.

No se puede desconocer que la empresa que maneja la plataforma establece en la mayoría de casos las condiciones del servicio, determina el monto a cobrar al usuario y porcentaje que le corresponde a la empresa, además de generar un registro de desempeño y establecer sanciones, lo cual llama la atención de forma particular debido a que las empresas niegan cualquier vínculo de relación laboral y denominan a sus trabajadores como "contratistas independientes". 22

Un criterio que se puede desprender de un análisis social y jurídico del derecho del trabajo, es que las empresas tergiversan la relación laboral típica y aparentan cumplir funciones de intermediación haciendo funcional la flexibilización, lo que les permite transferir los riesgos al trabajador, bajar costos y extenderse en el mercado conectando la oferta y la demanda de un servicio o producto, esto se traduce, en una aparente inestabilidad laboral, problemas en la seguridad y salud de los trabajadores considerando la inexistencia de obligaciones legales, falta de supervisión y formación, la rotación y duración de horarios de trabajo; y, por supuesto, una alerta para el sostenimiento del sistema de seguridad social. ${ }^{23}$

El aumento de esta modalidad genera controversia sobre la relación jurídica existente, debido a que la aparente inexistencia de la relación laboral dificulta la protección de derechos laborales, la exigibilidad de garantías que se relacionan a las condiciones típicas del empleo como protección social y condiciones saludables de trabajo, y también genera conflicto a nivel de control fiscal y competencia desleal.

En este sentido se recomienda el establecimiento de una nueva categoría de trabajo que considere las particularidades de las plataformas digitales y el establecimiento de mecanismos que garanticen progresivamente el acceso a la seguridad social, especialmente las relacionadas con la seguridad y salud en el trabajo, además de un sistema adecuado de control administrativo, tributario y laboral. ${ }^{24}$ Lo indispensable es que las transformaciones tecnológicas vayan de la mano de los cambios sociales, pero no solo aquello, sino que deben estar en el marco de la pertinencia jurídica que viabiliza la protección de los derechos.

22. Madariaga et al., Economía de plataformas y empleo ¿Cómo es trabajar para una app en Argentina?

23. Christoph Ernst y Verónica Robert, Cambio tecnológico y futuro del trabajo. Competencias laborales y habilidades colectivas para una nueva matriz productiva en Argentina (Buenos Aires: Oficina de País de la OIT para la Argentina, 2019).

24. Madariaga et al., Economía de plataformas y empleo ¿Cómo es trabajar para una app en Argentina? 


\section{PROBLEMÁTICAS JURÍDICAS EN EVIDENCIA: TRANSFORMACIONES PRODUCTIVAS Y TECNOLÓGICAS EN LOS ESTADOS CONSTITUCIONALES DE DERECHOS}

Toda vez que se ha dejado sentado la relación bilateral y recíproca entre cambio social y transformaciones tecnológicas; y, de esta última con las transformaciones o cambios productivos en un contexto de trabajo típico y atípico, es prudente realizar un abordaje ilustrativo de un espectro genérico de problemáticas jurídicas que podrían presentarse.

En el desarrollo de este apartado se hará énfasis en tres segmentos: primero, generalidades sobre el modelo político de Estado en el Ecuador y su implicación; segundo, ejemplificación de algunas garantías constitucionales como medios para el goce efectivo de los derechos; $\mathrm{y}$, tercero, una contextualización ilustrativa de las problemáticas jurídicas asociadas a las transformaciones tecnológicas, productivas y sociales.

Primero, el modelo político de Estado Constitucional de Derechos lleva consigo la carga del Neoconstitucionalismo o Nuevo Constitucionalismo Latinoamericano, lo que cambia la forma de comprender la Teoría del Derecho, la Teoría Democrática y la Teoría del Estado, por lo cual se tiene un considerable conjunto de elementos que diferencian a este modelo político de Estado frente al anterior denominado Estado de Derecho o Estado legalista. ${ }^{25}$

Hacer una descripción detallada del paradigma constitucional del Ecuador desbordaría el objeto de este estudio, por lo cual se hará una breve síntesis de lo más relevante. Se puede afirmar que, en este modelo de Estado, la autoridad pasa a ubicarse en un lugar secundario, esto debido a que la Constitución, como los derechos, son límites al poder y ninguna autoridad está por encima.

El Estado pasa a ser estructura de un sistema que sirve como medio para el goce efectivo de los derechos, su protección, exigibilidad y justiciabilidad en caso de que hayan sido vulnerados y necesiten de una reparación integral. ${ }^{26}$

25. Miguel Carbonell, "El neoconstitucionalismo en su laberinto", en Teoría del neoconstitucionalismo: ensayos escogidos (Madrid: Trotta, 2007); Roberto Gargarella y Christian Courtis, El nuevo constitucionalismo latinoamericano: promesas e interrogantes (Santiago: CEPAL, 2009); Roberto Viciano Pastor y Rubén Martínez Dalmau, “Aspectos generales del nuevo constitucionalismo latinoamericano”, en Política, justicia y Constitución, Crítica y Derecho, 2 (Quito: Corte Constitucional del Ecuador, 2012), 157-88; Ramiro Ávila, "Ecuador Estado Constitucional de Derechos y Justicia", en Constitución del 2008 en el contexto andino. Análisis de la doctrina y el derecho comparado, Justicia y Sociedad 3 (Quito: Ministerio de Justicia y Derechos Humanos, 2008), 29-37.

26. Jorge Calderón Gamboa, La reparación integral en la jurisprudencia de la Corte Interamericana de De- 
Por otra parte, la Constitución se transforma en la norma jurídica suprema del ordenamiento jurídico, donde los enunciados sustantivos (derechos) son de aplicación directa e inmediata. ${ }^{27}$

Otro elemento sustancial se refiere a la superación de la Teoría del Derecho Subjetivo, sobre la titularidad y legitimación activa en garantías jurisdiccionales, puesto que se incorpora la categoría sujetos de derechos, por la cual se incrementa el grado de exigibilidad. ${ }^{28}$ Tanto así que cualquier sujeto de manera individual, colectiva o las dos al mismo tiempo, o indistintamente, pueden activar garantías jurisdiccionales cuando se conozcan de la vulneración de derechos. Se agrega que la naturaleza es sujeto de derechos. ${ }^{29}$

Este salto cualitativo y cuantitativo también se ha visto representado en la conclusión diacrónica de la historia de la humanidad cuando unos individuos ejercían una relación de poder y subordinación sobre otros tratándolos como objetos. Aquí el ejemplo que permite hacer un análisis del trabajo y del trabajador en un contexto de transformaciones tecnológicas y productivas que atraviesan el fenómeno jurídico.

Los trabajadores en la historia han sido explotados como esclavos, y quizá esta relación ha ido evolucionando, sin dejar de lado quien tiene mayor capacidad de poder en esa relación. Por ilustrar este punto: se fue esclavo, se fue siervo, se es proletario, se es trabajador "asalariado".

Aunque las denominaciones resulten eufemismos que encubren la explotación del hombre por el hombre, o, en otras palabras, del ser por el ser, se les hace imposible permanecer por mucho tiempo ocultas.

La relación cíclica de las transformaciones tecnológicas, productiva y sociales vuelven necesario un marco constitucional de acciones positivas y negativas, no solo en lo jurídico, sino también en lo institucional y en políticas públicas.

Los cambios acelerados de las sociedades en todo ámbito pueden resultar muy positivos, pero también traer riesgos como anteriormente se ejemplifica sobre el trabajo atípico, y más común en el trabajo típico como categoría de la habitual relación de dependencia en contextos de empresas tradicionales.

rechos Humanos: estándares aplicables al nuevo paradigma mexicano (Ciudad de México: Instituto de Investigaciones Jurídicas, 2013).

27. Ricardo Guastini, "La constitucionalización del ordenamiento jurídico: el caso italiano", en Neoconstitucionalismo(s), ed. Miguel Carbonell (Madrid: Trotta, 2003).

28. Entiéndase el incremento de exigibilidad de los derechos como la oportunidad que tienen los sujetos de derechos de poder ejercer acciones que se ventilarán por el ámbito jurisdiccionales, sin necesidad de ser representados por alguien más sino por sus propios derechos.

29. Diana Murcia, "El sujeto naturaleza: elementos para su comprensión", en Naturaleza con derechos: de la filosofia a la política (Quito: Abya-Yala, 2011), 287-317. 
Toda vez que estas transformaciones avanzan, comienza una nueva exigencia para el ser humano, volviéndolo más vulnerable al perfil de las decisiones del Estado a través del gobierno y de los empleadores. Hay que recordar el primer punto sobre la necesidad de tener un Estado Constitucional de Derechos, puesto que una de sus principales finalidades es frenar el abuso de poder.

La Constitución está diseñada para evitar que ante estos cambios - algunos bruscos y otros prolongados - se reduzca el riesgo de vulnerabilidad de los trabajadores y trabajadoras de que puedan incrementarse sus horas de trabajo, se generen despidos injustificados, se aproveche de la necesidad de la persona trabajadora, se discrimine por el grado de conocimiento, entre otros.

Para afrontar aquello ha de entenderse que no pueden existir derechos sin garantías, y la Constitución define tres tipos — aunque puedan existir muchas más garantías constitucionales $^{30}$-: 1) garantías normativas; 2) garantías de políticas públicas; y 3) garantías jurisdiccionales. ${ }^{31}$ Se incrementa el catálogo de garantías en correlación con el aumento del catálogo de derechos, por lo tanto, de este ejercicio se pretende la disminución de toda práctica de abuso de poder que intente vulnerar derechos. Para los trabajadores esto es de vital importancia, y en las siguientes líneas se ilustrará el rol que la Constitución asumiría ante una eventual o abrupta presencia de las tecnologías en el ámbito del trabajo.

Finalmente, queda ilustrar los anteriores apartados en una contextualización de transformaciones tecnológicas, productivas y sociales. Así, se tomará como referencia la actual situación del país: ${ }^{32}$ estados de excepción, estados de emergencia, crisis sanitaria, pandemia Covid-19, entre otros factores.

Para el sector del trabajo, esta crisis ha significado en la práctica lo que en apartados anteriores se menciona como teoría: a) las transformaciones suceden a todo nivel y establecen nuevas relaciones que actúan de manera bilateral; b) las exigencias sobre las personas se vuelven imperativas e inesperadas; c) Estado, mercado y sociedad tampoco se encuentran totalmente preparados; y d) se evidencia improvisación a toda esfera y con ello afectación a ciertos derechos. A todo esto puede sumarse un impacto obligatorio de nuevas modalidades de trabajo, uso de plataformas tecnológicas, y trabajo desde los hogares.

30. La clasificación de garantías constitucionales puede ser más amplia, por ejemplo, referirse a garantías primeras, secundarias, abstractas, concretas, institucionales, sociales, etc. Para este escrito solo se ha tomado en cuenta la clasificación explícita de la Constitución de la República del Ecuador de 2008.

31. Ecuador, Constitución de la República del Ecuador, Registro Oficial 449, Suplemento, 20 de octubre de 2008 , arts. 84 y ss.

32. Se deja a salvo el ejercicio ilustrativo en diversas y múltiples situaciones para ser abordadas en futuros trabajos académicos que traten las mismas variables o tomen como referencia el esquema propuesto. 
El cambio social debido a la pandemia trae por consecuencia transformaciones tecnológicas como el teletrabajo que implica un tipo de transformación productiva, como el quiebre en el giro del negocio de una empresa o el cambio a la matriz productiva de un Estado. Si no se toma en cuenta esta dinámica, será muy difícil tomar decisiones políticas y legislar de manera adecuada sin afectar los derechos de los trabajadores; por ejemplo:

1. Si se deja a voluntad de las partes los acuerdos sobre la forma de trabajo, jornadas y remuneraciones, se estaría desconociendo que la persona trabajadora está en desventaja desde un principio en esa relación de poder.

2. Si no se regula adecuadamente el teletrabajo puede correrse el riesgo que se afecte estructuralmente la dinámica de la sociedad, debido al uso del tiempo de trabajo. Si no se establecen prohibiciones o protecciones para el disfrute del tiempo libre, familia, recreación, entre otros, se terminará por tener una sociedad instrumentalizada al trabajo y encerrada en sus hogares, pendientes a toda hora de las actividades laborales. Es importante hablar aquí del derecho a la desconexión como derivado del derecho al descanso y enlazado con el proyecto de vida.

3. Si no se controlan las plataformas virtuales "colaborativas" no por su crecimiento sino por el impacto social, laboral y jurídico, se tendrán, por una parte, trabajadores sin protección social, empresas que se benefician de elevados porcentajes y una legislación que permite la salida de capitales, además de un régimen impositivo poco útil sobre ese tipo de generación de riqueza.

4. Si las personas trabajadoras no se instruyen de manera óptima en la mecánica de las transformaciones tecnológicas y productivas de su sector, también representaría un impacto en la economía del país y de la empresa, para lo cual, debe garantizarse la educación o, más bien, incrementarse gradualmente el presupuesto destinado para el sector educativo, tecnológico y de investigación.

5. Si el gobierno comienza a ceder a las pretensiones de los intereses de grupos de poder, habrá un ejercicio de la política abusivo en la toma de decisiones y, por lo tanto, utilizará sus potestades como la de creación de normas a través de decretos, acuerdos, resoluciones, entre otros, para responder a esos intereses, aún sin tener legitimidad absoluta. Esto aumentaría las desigualdades, y que unos sean más afectados que otros.

Estos son algunos ejemplos ilustrativos del espacio de transformaciones sociales, económicas, productivas y tecnológicas, pero ¿qué se puede hacer al respecto? Para los conflictos jurídicos que nacen del abuso de poder de empleadores para firmar acuerdos que reducen los derechos ha de recordárseles que los principios de aplicación de los derechos son mandatos de optimización y que deben ser observados por 
ellos y, además, en caso de llegar a una autoridad jurisdiccional, formar parte de su motivación en la decisión. No se puede alegar falta de norma y desconocer los derechos. $^{33}$

Ante conflictos de ley, colisión de derechos, lagunas normativas y axiológicas, ${ }^{34} \mathrm{se}$ ha de resolver siempre con el sentido o significados primigenios de la Constitución, no puede quedarse una persona sin recibir justicia, aun quedando la posibilidad de verificar la mejor situación de los derechos en el derecho internacional de los derechos humanos. ${ }^{35}$

La creación de normas no puede ser contraria a la Constitución y debe gozar no solo de validez formal sino también de validez material que busca un impacto positivo, justo y equitativo en la sociedad, aun si es creada por quienes tienen potestad legislativa, debería considerarse fuera del ordenamiento jurídico cuando es evidentemente injusta.

Para finalizar con este apartado, las garantías jurisdiccionales deben siempre estar a disposición de la población para la exigibilidad y justiciabilidad de sus derechos, así como a recibir una reparación integral. En último caso, recurrir a su derecho a la resistencia si es que la institucionalidad, el gobierno, entre otros actores, han llevado a un Estado que explota al ser por el ser. ${ }^{36}$

\section{CONCLUSIONES}

Se ha demostrado que las relaciones entre los cambios tecnológicos, productivos y sociales presentan un tipo de relación cíclica, por una parte, que se seguirán reproduciendo a lo largo de la historia de la humanidad, y, por otra, que suceden en un sentido

33. Ibíd., art. 11.

34. Josep Aguiló Regla, “Lagunas constitucionales”, sn, n. ${ }^{\circ} 1$ (2019): 19; Luis Prieto Sanchís, Derechos fundamentales, neoconstitucionalismo y ponderación judicial (Lima: Palestra Editores, 2007); Manuel Atienza, El Derecho como Argumentación (Barcelona: Ariel, 2006).

35. Danilo Alberto Caicedo Tapia, "El bloque de constitucionalidad en el Ecuador. Derechos Humanos más allá de la Constitución", Foro: Revista de Derecho, n. ${ }^{\circ} 12$ (II semestre 2009): 5-30; Vanessa Suelt-Cock, "El bloque de constitucionalidad como mecanismo de interpretación constitucional. Aproximación a los contenidos del bloque en derechos en Colombia", Vniversitas 65, n. ${ }^{\circ} 133$ (2016): 301.

36. Merino Arza y Darwin Jorge, "El derecho de la resistencia y la ausencia de normativa en el Estado Constitucional de derechos y justicia". 2015; Judith Alejandra Carrillo, "El Derecho a la Resistencia en la Constitución de la República del Ecuador" (Universidad de las Américas, 2010); Milton Rocha, "El rol de la creatividad en la resistencia más allá de un derecho: las actuales resistencias contra la explotación de recursos naturales en el Ecuador", en Refundación del constitucionalismo social. Reflexiones a los cien años de la Constitución de Querétaro, ed. Claudia Storini (Quito: Universidad Andina Simón Bolívar, Sede Ecuador / Corporación Editora Nacional, 2019). 
de correspondencia o bidireccionalidad, porque los efectos o impactos no solo vienen de un solo tipo de cambio, sino que acontecen intermitentemente. Tanto pueden incidir las transformaciones tecnológicas a las productivas y viceversa; como pueden los cambios sociales incidir en las dos anteriores y también a la inversa.

El efecto de la aplicación de las nuevas tecnologías sobre la producción y su impacto en el empleo es distinto en cada etapa histórica: la dinámica económica y social no responde de la misma forma ante los desafíos que se le puedan presentar, en el contexto actual de la tecnología avanza a gran velocidad y su impacto genera efectos a nivel global, a diferencia de etapas anteriores en las que su alcance se presenta de forma gradual.

La velocidad y el alcance con que las actuales innovaciones tecnológicas están cambiando la dinámica social, incluido el mercado laboral, genera incertidumbre. Algunas teorías señalan que las transformaciones tecnológicas inciden en la medida de que destruyen ciertas actividades ya sea rutinarias o cognitivas en un mayor o menor grado y a su vez generan la necesidad de redefinir o crear nuevas ocupaciones.

Las nuevas ocupaciones requieren ciertos tipos de perfiles que abren campo a la polarización del empleo perfiles altamente cualificados con salarios altos o de baja cualificación con salarios bajos, generando gran tensión y desigualdad, lo que invita a pensar que el manejo del conocimiento, en la actual revolución será la clave para ubicarse en un contexto u otro.

Se verifica la necesidad del Estado Constitucional de Derechos para evitar los abusos de poder y el desequilibrio de las relaciones para evitar el incremento de las desigualdades debido a la transformaciones sociales, económicas, políticas, tecnológicas y productivas en el ámbito del trabajo.

\section{BIBLIOGRAFÍA}

Arntz, Melanie, Gregory Terry, y Ulrich Zierahn. "The Risk of Automation for Jobs in oecd Countries: A Comparative Analysis". Employment and Migration Working Papers, n. ${ }^{\circ} 186$ (2016): 1-33. https://url2.cl/32iIa.

Arza, Merino, y Darwin Jorge. "El derecho de la resistencia y la ausencia de normativa en el Estado Constitucional de derechos y justicia". 2015.

Atienza, Manuel. El Derecho como argumentación. Barcelona: Ariel, 2006.

Ávila, Ramiro. "Ecuador Estado Constitucional de Derechos y Justicia". En Constitución del 2008 en el contexto andino. Análisis de la doctrina y el derecho comparado, 29-37. Justicia y Sociedad 3. Quito: Ministerio de Justicia y Derechos Humanos, 2008.

Bouza, Fermín. "Innovación tecnológica y cambio social". En Las encrucijadas del cambio social, Universidad de Vigo, 85-97. Vigo: Servizo de Publicacións, 2002. 
Caicedo Tapia, Danilo Alberto. "El bloque de constitucionalidad en el Ecuador. Derechos Humanos más allá de la Constitución". Foro: Revista de Derecho, n. ${ }^{\circ} 12$ (II semestre 2009): 5-30.

Calderón Gamboa, Jorge. La reparación integral en la jurisprudencia de la Corte Interamericana de Derechos Humanos: estándares aplicables al nuevo paradigma mexicano. Ciudad de México: Instituto de Investigaciones Jurídicas, 2013.

Carbonell, Miguel. "El neoconstitucionalismo en su laberinto". En Teoría del neoconstitucionalismo: ensayos escogidos. Madrid: Trotta, 2007.

Carrillo, Judith. "El Derecho a la Resistencia en la Constitución de la República del Ecuador". Universidad de las Américas, 2010.

Catalano, Ana. Tecnología, innovación y competencias ocupacionales en la sociedad del conocimiento. Documentos de Trabajo 22. Argentina: Oficina de la OIT para la Argentina, 2018. https://url2.cl/MtVcH.

Doménech, Rafael, José García, Mirian Montañez, y Alejandro Neut. “Afectados por la revolución digital: El caso de España”. Papeles de Economía Española, 2018, 128-45.

Ecuador. Constitución de la República del Ecuador. Registro Oficial, Suplemento 449, 20 de octubre de 2008.

Ernst, Christoph, y Verónica Robert. Cambio tecnológico y futuro del trabajo. Competencias laborales y habilidades colectivas para una nueva matriz productiva en Argentina. Buenos Aires: Oficina de la OIT para la Argentina, 2019.

Ford, Martin. El auge de los robots: La tecnología y la amenaza de un futuro sin empleo. Barcelona: Paidós Ibérica, 2016.

Frey, Carl, y Michael Osborne. "The future of employment: How susceptible are jobs to computerisation?”. Technological Forecasting \& Social Change 49 (2017): 254-80. https:// url2.cl/5z9Rs.

Gargarella, Roberto, y Christian Courtis. El nuevo constitucionalismo latinoamericano: promesas e interrogantes. Santiago: CEPAL, 2009.

Garza, Enrique. La formación socioeconómicas neoliberal: debates teóricos acerca de la reestructuración de la producción y evidencia empírica para América Latina. Primera. Plaza y Valdés, 2001.

Guastini, Ricardo. "La constitucionalización del ordenamiento jurídico: el caso italiano”. En Neoconstitucionalismo(s), editado por Miguel Carbonell. Madrid: Trotta, 2003.

Lais, Abramo, y Salazar. "El trabajador frente a la automatización: efectos sociales y percepción de los trabajadores". Revista Mexicana de Sociología 50 (1988): 61-9.

Lück, Esther Hermes. "El proceso de transformación tecnológica y la formación docente”. Revista de Universidad y Sociedad del Conocimiento 6, n. ${ }^{\circ} 1$ (2009): 1-10. https://n9.cl/b0bw.

Madariaga, Javier, Cesar Buenadicha, Erika Molina, y Christoph Ernst. Economía de plataformas y empleo ¿Cómo es trabajar para una app en Argentina? Buenos Aires: CIPPEC-BID / OIT, 2009. 
Martínez, Daniel. "Sobre el incierto futuro del trabajo y del rol de los actores sociales". Revista Economía 41, n. ${ }^{\circ} 81$ (2018): 69-100. https://url2.cl/sDI8y.

Marx, Karl. El Capital. Traducido por Isaac Zeiger. Buenos Aires: Gradifco, Pensadores Universales, 2006.

Murcia, Diana. "El sujeto naturaleza: elementos para su comprensión”. En Naturaleza con derechos: de la filosofia a la política, 287-317. Quito: Abya-Yala, 2011.

OIT. "El empleo atípico en el mundo: Retos y perspectivas. Presentación resumida del informe". Ginebra: Oficina Internacional del Trabajo, 2016.

Prieto Sanchís, Luis. Derechos fundamentales, neoconstitucionalismo y ponderación judicial. Lima: Palestra, 2007.

Real Academia Española. Diccionario de la lengua española. RAE, 2014. https://dle.rae.es.

Regla, Josep Aguiló. “Lagunas constitucionales”. sn, n. ${ }^{\circ} 1$ (2019): 19.

Rifkin, Jeremy. El fin del trabajo: Nuevas tecnologías contra puestos de trabajo. Nueva York: Paidós Ibérica, 1996.

Rocha, Milton. "El rol de la creatividad en la resistencia más allá de un derecho: las actuales resistencias contra la explotación de recursos naturales en el Ecuador". En Refundación del constitucionalismo social. Reflexiones a los cien años de la Constitución de Querétaro. Quito: Corporación Editora Nacional, 2019.

Roitter, Sonia. Cambio tecnológico y empleo: Aportes conceptuales y evidencia frente a la dinámica en curso. Buenos Aires: Centro Interdisciplinario de Estudios en Ciencia, Tecnología e Innovación, 2019.

Suelt-Cock, Vanessa. "El bloque de constitucionalidad como mecanismo de interpretación constitucional. Aproximación a los contenidos del bloque en derechos en Colombia". Vniversitas $65,{ }^{\circ}{ }^{\circ} 133$ (2016): 301.

Viciano Pastor, Roberto, y Rubén Martínez Dalmau. “Aspectos generales del nuevo constitucionalismo latinoamericano”. En Política, justicia y Constitución, 157-88. Crítica y Derecho, 2. Quito: Corte Constitucional del Ecuador, 2012. 